\title{
TOEPLITZ OPERATORS IN SEGAL-BARGMANN SPACES OF VECTOR-VALUED FUNCTIONS
}

\author{
DARIUSZ CICHOŃ and HAROLD S. SHAPIRO*
}

\begin{abstract}
We discuss new results concerning unbounded Toeplitz operators defined in Segal-Bargmann spaces of (vector-valued) functions, i.e. the space of all entire functions which are square summable with respect to the Gaussian measure in $\mathrm{C}^{n}$. The problem of finding adjoints of analytic Toeplitz operators is solved in some cases. Closedness of the range of analytic Toeplitz operators is studied. We indicate an example of an entire function inducing a Toeplitz operator, for which the space of polynomials is not a core though it is contained in its domain.
\end{abstract}

\section{Introduction}

We begin by introducing basic definitions and notations. Let $\mathscr{H}$ be a complex separable Hilbert space. Consider $L^{2}(\mu) \otimes \mathscr{H}$, the Hilbert space of all complex Borel functions taking values in $\mathscr{H}$ which are square-integrable on $\mathrm{C}^{n}$ with respect to the measure $\mu$ given by $d \mu(z)=\pi^{-n} e^{-z \cdot \bar{z}} d V(z)$, where $V$ is the Lebesgue measure in $\mathrm{C}^{n}$ and $z \cdot \bar{z}=\left|z_{1}\right|^{2}+\cdots+\left|z_{n}\right|^{2}$ for $z=\left(z_{1}, \ldots, z_{n}\right) \in \mathrm{C}^{n}$. The inner product in $L^{2}(\mu) \otimes \mathscr{H}$ is given by

$$
\langle f, g\rangle_{(\mathscr{H})}=\int_{\mathrm{C}^{n}}\langle f(\zeta), g(\zeta)\rangle_{\mathscr{H}} d \mu(\zeta), \quad f, g \in L^{2}(\mu) \otimes \mathscr{H},
$$

where $\langle\cdot, \cdot\rangle_{\mathscr{C}}$ stands for the inner product in $\mathscr{H}$. The norm induced by the above defined inner product is denoted by $\|\cdot\|_{(\mathscr{H})}$ and in case $\mathscr{H}=\mathrm{C}^{N}$ by $\|\cdot\|_{(N)}$. The Segal-Bargmann space $\mathscr{B}_{n} \otimes \mathscr{H}$ (abbreviated $\mathscr{B} \otimes \mathscr{H}$ ) is a closed subspace of $L^{2}(\mu) \otimes \mathscr{H}$ composed of all entire functions belonging to $L^{2}(\mu) \otimes \mathscr{H}$. By $P \otimes I_{\mathscr{H}}$ we mean the orthogonal projection of $L^{2}(\mu) \otimes \mathscr{H}$ onto $\mathscr{B} \otimes \mathscr{H}$. We will use the following identifications: $L^{2}(\mu)=L^{2}(\mu) \otimes \mathrm{C}, \mathscr{B}=\mathscr{B} \otimes \mathrm{C}$, $\langle\cdot, \cdot\rangle=\langle\cdot, \cdot\rangle_{(1)},\|\cdot\|=\|\cdot\|_{(1)}$ and $P=P \otimes I_{\mathrm{C}}$. Observe that $\mathscr{B} \otimes \mathrm{C}^{N}$ can be identified with $\mathscr{B} \oplus \ldots \oplus \mathscr{B}$ (N-times), and following this we have $\mathscr{B} \otimes C^{N}=$ $\left\{\left(f_{1}, \ldots, f_{N}\right): f_{1}, \ldots, f_{N} \in \mathscr{B}\right\}$ and $\left\|\left(f_{1}, \ldots, f_{N}\right)\right\|_{(N)}^{2}=\int_{C^{n}}\left(\left|f_{1}\right|^{2}+\cdots+\right.$ $\left.\left|f_{N}\right|^{2}\right) d \mu$.

\footnotetext{
* The first named author is supported by KBN under grant no. 2 P03A 00417.
}

Received October 1, 2001; in revised form June 4, 2002. 
Given $f: \mathrm{C}^{n} \rightarrow \mathrm{C}$ and $h \in \mathscr{H}$ we define $(f \otimes h)(z):=f(z) h, z \in \mathrm{C}^{n}$. Put $e_{a}(z):=e^{z \cdot \bar{a}}$, where $z \cdot a:=\sum_{k=1}^{n} z_{k} a_{k}$ and $\bar{a}:=\left(\bar{a}_{1}, \ldots, \bar{a}_{n}\right)$ for $a=\left(a_{1}, \ldots, a_{n}\right) \in \mathrm{C}^{n}$ and $z=\left(z_{1}, \ldots, z_{n}\right) \in \mathrm{C}^{n}$. Denote by $\mathscr{E}\left(=\mathscr{E}_{n}\right)$ the linear span of the set of functions $\left\{e_{a}: a \in \mathrm{C}^{n}\right\}$. It can be checked that $\langle f(z), h\rangle_{\mathscr{H}}=\left\langle f, e_{z} \otimes h\right\rangle_{(\mathscr{H})}$ for $f \in \mathscr{B} \otimes \mathscr{H}, h \in \mathscr{H}$ and $z \in \mathrm{C}^{n}$ (cf. [4]), which is referred to as the reproducing property for $\mathscr{B} \otimes \mathscr{H}$. By $\mathscr{P}\left(=\mathscr{P}_{n}\right)$ we denote the space of all analytic polynomials in $C^{n}$. Both $\mathscr{P}$ and $\mathscr{E}$ are dense subsets of $\mathscr{B}$. The sequence $f_{k}(z):=z^{k} / \sqrt{k !}, k \in \mathbf{N}^{n}, z \in \mathrm{C}^{n}$, forms an orthonormal basis for $\mathscr{B}$, where according to the standard multiindex notation $z^{k}:=z_{1}^{k_{1}} \cdot \ldots \cdot z_{n}^{k_{n}}, k !:=k_{1} ! \cdot \ldots \cdot k_{n}$ ! and $|k|:=k_{1}+\ldots+k_{n}$ for all $z=\left(z_{1}, \ldots, z_{n}\right) \in \mathrm{C}^{n}$ and $k=\left(k_{1}, \ldots, k_{n}\right) \in \mathbf{N}^{n}(\mathbf{N}=\{0,1,2, \ldots\})$.

$A$ is called a linear operator in a complex Hilbert space $\mathscr{H}$ if its domain $\mathscr{D}(A)$ is a linear subspace of $\mathscr{H}$ and $A: \mathscr{D}(A) \rightarrow \mathscr{H}$ is a linear mapping. As usual, $A^{*}, \bar{A}, \mathscr{R}(A)$ stand for the adjoint (provided it exists), the closure of $A$ and the range of $A$, respectively. We say that a linear subspace $\mathscr{D} \subseteq \mathscr{D}(A)$ is a core for a closable operator $A$ if $\left(\left.A\right|_{\mathscr{D}}\right)^{-}=\bar{A}$. All these notions are also meaningful for linear operators acting between different Hilbert spaces. By $B(\mathscr{H}, \mathscr{K})(\mathscr{H}, \mathscr{K}$-Hilbert spaces) we denote the space of all bounded linear operators $A: \mathscr{H} \rightarrow \mathscr{K}$, and $B(\mathscr{H}):=B(\mathscr{H}, \mathscr{H})$. The graph norm $\|\cdot\|_{A}$ is defined by $\|f\|_{A}^{2}=\|f\|_{\mathscr{H}}^{2}+\|A f\|_{\mathscr{H}}^{2}, f \in \mathscr{D}(A)$.

Write $\mathscr{P} \otimes \mathscr{H}$ for the space of polynomials (in $\mathrm{C}^{n}$ ) taking values in $\mathscr{H}$, i.e. the totality of functions of type $\sum_{j=0}^{K} p_{j} \otimes h_{j}$, where $p_{j} \in \mathscr{P}_{n}, h_{j} \in \mathscr{H}$ and $K \in \mathrm{N}$ (in the case where $\mathscr{H}=\mathrm{C}^{N}$ the elements of this space are identified with $N$-tuples of polynomials). Spaces $\mathscr{E} \otimes \mathscr{H}$ and $\mathscr{P} \otimes B(\mathscr{H}, \mathscr{K})$ are defined analogously. Observe that $p \in \mathscr{P} \otimes B\left(\mathrm{C}^{M}, \mathrm{C}^{N}\right)$ can be written as a matrix $\left[p_{k l}\right]_{k=1, \ldots, N}^{l=1, \ldots, M}$ with some polynomials $p_{k l} \in \mathscr{P}$. Given $p \in \mathscr{P} \otimes B(\mathscr{H}, \mathscr{K})$, $p(z)=\sum_{|j|<K} A_{j} z^{j}, A_{j} \in B(\mathscr{H}, \mathscr{K}), z \in \mathrm{C}^{n}$, define $p^{\#} \in \mathscr{P} \otimes B(\mathscr{K}, \mathscr{H})$ via $p^{\#}(z)=p(\bar{z})^{*}, z \in C^{n}$, and a differential operator $(p(D) F)(z)=$ $\sum_{|j|<K} A_{j} D^{j} F(z), z \in C^{n}$, where $F: C^{n} \rightarrow \mathscr{H}$ is an analytic function (here $D^{j}=\frac{\partial^{|j|}}{\partial z_{1}^{j_{1}} \ldots \partial z_{n}^{j_{n}}}$ for $j=\left(j_{1}, \ldots, j_{n}\right)$ ). We will regard $p(D)$ as an operator in $\mathscr{B} \otimes \mathscr{H}$ and its domain is defined as $\mathscr{D}(p(D))=\{F \in \mathscr{B} \otimes \mathscr{H}$ : $p(D) F \in \mathscr{B} \otimes \mathscr{K}\}$.

Let $\varphi: C^{n} \rightarrow B(\mathscr{H}, \mathscr{K})$ be an analytic function. Define $\varphi^{\#}(z):=\varphi(\bar{z})^{*}$, which amounts to taking adjoints of all coefficients in the series expansion of $\varphi$, and $\varphi^{*}(z):=\varphi(z)^{*}$ for $z \in \mathrm{C}^{n}$. A Toeplitz operator ${ }^{1}$ with symbol $\varphi$ is defined by $\mathscr{D}\left(T_{\varphi}\right)=\{f \in \mathscr{B} \otimes \mathscr{H}: \varphi f \in \mathscr{B} \otimes \mathscr{K}\}$ and $T_{\varphi} f=\varphi f$ for $f \in \mathscr{D}\left(T_{\varphi}\right)$, where $(\varphi f)(z)=\varphi(z) f(z), z \in \mathrm{C}^{n}$. We need one more operator

\footnotetext{
${ }^{1}$ What we have defined here is, in fact, an analytic Toeplitz operator, cf. [4] for the general case.
} 
in $\mathscr{B} \otimes \mathscr{H}$ denoted by $\Pi_{\varphi^{*}}$, which is defined via

$$
\Pi_{\varphi^{*}} f(z)=\int_{\mathrm{C}^{n}} \varphi^{*}(\zeta) f(\zeta) e^{z \cdot \zeta} d \mu(\zeta), \quad z \in \mathrm{C}^{n},
$$

and its domain consists of all $f: \mathrm{C}^{n} \rightarrow \mathscr{K}$ such that the above integral exists ${ }^{2}$ for all $z \in \mathrm{C}^{n}$ and the function in $z$ defined by it lies in $\mathscr{B} \otimes \mathscr{H}$. Since $\Pi_{p^{*}}=$ $p^{\#}(D)$ for $p \in \mathscr{P} \otimes B(\mathscr{H}, \mathscr{K})$ (cf. [4]), we may regard $\Pi_{\varphi^{*}}$ as a generalized differential operator. It is known that $\Pi_{p^{*}}=\left(\left.T_{p}\right|_{\mathscr{E} \otimes \mathscr{H}}\right)^{*}=\left(T_{p} \mid \mathscr{P} \otimes \mathscr{H}\right)^{*}$ (cf. [4, Corollary 7.3]). The following abstract lemma reflects the connection between $T_{p}$ and $\Pi_{p^{*}}$.

Lemma 1.1. Let $A$ be a closed operator in $\mathscr{H}$ and $\mathscr{V} \subseteq \mathscr{D}(A)$ be a dense subspace of $\mathscr{H}$. Then for $B:=\left(\left.A\right|_{\mathscr{V}}\right)^{*}$ the following conditions are equivalent:

(i) $A^{*}=B$;

(ii) $\operatorname{ker}(I+A B)=\{0\}$;

(iii) $A B$ is symmetric;

(iv) $A B$ is non-negative;

(v) $A B$ is selfadjoint;

(vi) $\mathscr{V}$ is a core for $A$.

Interchanging $A$ and $B$ in (i)-(v) gives another condition equivalent to (vi).

Proof. Observe that $A^{*} \subseteq B$ and $B$ is closed. Both these facts are in frequent use in the following proof. We begin with (i) $\Leftrightarrow($ ii). Let $\Gamma(A)$ denote the graph of $A$, i.e. the set $\{(x, y): x \in \mathscr{D}(A), A x=y\}$. Then one can easily show that $\Gamma(B) \ominus \Gamma\left(A^{*}\right)$ consists of all $(x, y)$ such that $x \in \mathscr{D}(B)$, $y=B x \in \mathscr{D}(A)$ and $x+A y=0$, which immediately implies the desired equivalence. Equivalence (i) $\Leftrightarrow$ (vi) is implied by taking adjoints in (i). By standard operator theory (i) implies (iii), (iv) and (v). Since each of (iv) and (v) implies (iii) by definition, it suffices to prove implication (iii) $\Rightarrow$ (i). Observe that $A A^{*}$ is a selfadjoint operator contained in $A B$. Hence by (iii) $A A^{*}=A B$, which implies (ii) and, consequently, (i). So the equivalence of conditions (i)-(vi) is established.

Condition (i) may be rewritten as $A=B^{*}$. Thus the last statement of the assertion follows.

The adjointness hypothesis consists in equality $T_{p}^{*}=\Pi_{p^{*}}$ for $p \in \mathscr{P} \otimes$ $B(\mathscr{H})$, which was proved in some cases, e.g. $p \in \mathscr{P}_{n}$, cf. [10], or $p \in \mathscr{P}_{1} \otimes$ $B(\mathscr{H})$ and its leading coefficient is surjective, cf. [4]. In the present paper we present new results concerning the hypothesis.

\footnotetext{
${ }^{2}$ i.e. it is weakly convergent, cf. [4]
} 
Toeplitz operators of type $T_{p}$ as above, and their adjoints, play an important role in extending known results on partial differential operators and convolution operators in the space of entire functions to the context of SegalBargmann spaces. Suppose for simplicity we consider one-variable SegalBargmann space $\mathscr{B}$. In the classical theory of linear differential equations of infinite order, with constant coefficients (one variable), one starts with a "symbol function" $\varphi$, which is entire and of exponential type, and the thematic problem is to show that every entire function $u$ which satisfies the equation $\varphi(D) u=0$ is, in the sense of uniform approximation on compact sets, the limit of a sequence each term of which is a finite linear combination of "monomial exponential" solutions of this same equation, that is solutions of the form $m(z)=z^{r} \exp (w z)$, where $w \in \mathrm{C}$ and $r \in \mathbf{N}$. There are various ways, all equivalent, to define the operator $\varphi(D)$, for example as an infinite order differential operator, or as convolution with a certain compactly supported measure on $C$, whose Fourier-Laplace transform is $\varphi$. This theory originated in the study of difference and differential-difference equations, and there is a solid treatment of this e.g. [6], for more modern versions see e.g. [5].

A more refined question (arising from Delsarte's notion of mean periodic functions, originally in the context of functions of a real variable but extended to the holomorphic category by Laurent Schwartz and others) is this: Given $u$ entire such that its translates (equivalently its derivatives) fail to span $\mathrm{Hol}(\mathrm{C})$ (the entire functions), which is equivalent to the existence of some nontrivial $\varphi$ of exponential type such that $\varphi(D) u=0$, do those translates none the less span enough monomial exponentials to, in turn, span ("synthesize") $u$ ? This is the spectral synthesis question for $\mathrm{Hol}(\mathrm{C})$. It is known that this question too has an affirmative answer; in higher dimensions however there are counterexamples to the spectral synthesis version, whereas the positive solution to the versions in the preceding paragraph holds in all dimensions.

Using this as a guide, one can formulate analogous questions in other topological vector spaces of entire functions. For example, in $\mathscr{B}$ let us ask whether the solutions to $\varphi^{\#}(D) u=0$ are in the closure of the monomial exponential solutions. Here we may take as our definition of $\varphi^{\#}(D)$ the operator $\Pi_{\varphi^{*}}$. It is no longer necessary to suppose $\varphi$ of exponential type, we get a sensible problem whenever the product of $\varphi$ by each exponential is in $\mathscr{B}$; however, to fix ideas think first of the case where $\varphi$ is of exponential type; even here the question to be posed is still unsolved. The monomial exponentials in question consist precisely of, for each zero $w$ of $\varphi$, the functions $z^{k} \exp (\bar{w} z), k=0, \ldots, r-1$ where $r$ is the multiplicity of the zero.

The question whether these span all solutions to $\varphi^{\#}(D) u=0$ in the norm topology of $\mathscr{B}$, then translates, by duality, to this: suppose $h$ in $\mathscr{B}$ is orthogonal to the monomial exponentials belonging to $\operatorname{ker} \varphi^{\#}(D)$; this is equivalent to it 
vanishing on all zeros of $\varphi$, with the appropriate multiplicities, i.e. to the assertion: $h / \varphi$ is an entire function. We ask if $h$ must then be orthogonal to each $u$ that is, in turn, orthogonal to all $\varphi(z) \exp (t z)$ with $t \in \mathrm{C}$. Since $h=\varphi F$ for some entire $F$, the question is whether $\varphi F$ is approximable in $\mathscr{B}$-norm by exponential multiples of $\varphi$. And this is just the question: do the finite linear combinations of exponentials span every entire function, in the metric of $L^{2}\left(|\varphi|^{2} d \mu\right)$ ?

In [10] an affirmative solution was given for $\varphi$ which are exponential polynomials, and indeed in any number of variables. But, for other classes of entire functions $\varphi$ this is unsolved.

The counterexample of Borichev [1] (and our version of it below, in Section 4) are a borderline case: they can be said to disprove spectral synthesis for some "symbol function" $\varphi$ of very large growth - so large, indeed, that the equation $\varphi^{\#}(D) u=0$ has to be interpreted in a generalized sense, namely $u$ (in $\mathscr{B}$ ) is orthogonal to all the polynomial multiples of $\varphi$ (this is all we have to work with, since the exponential multiples, needed for the natural definition, do not lie in $\mathscr{B})$. So, it is of interest to know whether a corresponding counterexample exists where $\varphi$ is of smaller growth, at least multiplying exponentials into $\mathscr{B}$.

\section{Adjointness}

In what follows $\Delta(w, R) \subseteq \mathrm{C}^{n}$ stands for the open polydisk with radius $R$ centered at $w$. Let $P_{k}: \mathrm{C}^{n} \rightarrow \mathrm{C}, k=1, \ldots, n$, denote projection onto the $k$-axis, i.e. $P_{k}(z):=z_{k}$ for all $z=\left(z_{1}, \ldots, z_{n}\right) \in \mathrm{C}^{n}$.

Lemma 2.1. Let $X$ be a Borel measurable set such that $P_{k}(X)$ is bounded for some $k \in\{1, \ldots, n\}$ and $R:=\inf \left\{r>0: P_{k}(X)\right.$ is contained in $\Delta(w, r)$ with some $w\}$. Then there exists a constant $C$ depending only on $R$ such that

$$
\int_{C^{n}}|f|^{2} d \mu \leq C \int_{C^{n} \backslash X}|f|^{2} d \mu
$$

for all entire functions $f: \mathrm{C}^{n} \rightarrow \mathrm{C}$.

Proof. First consider the one-dimensional case. Fix $R>0, w \in \mathrm{C}$ and an entire function $f$ defined on C. Put $I(r):=\int_{0}^{2 \pi}\left|f\left(r e^{i t}+w\right)\right|^{2} d t, r \geq 0$. Since $I(r)$ is increasing with respect to $r$ we have

$$
r e^{-r^{2}} I(r) \leq(R+r) e^{3 R^{2}} e^{-(R+r)^{2}} I(R+r), \quad r \in[0, R] .
$$

Both sides of this inequality are functions in $r$, so integrating over the interval 
$[0, R]$ (with respect to the Lebesgue measure) yields

$$
\int_{\Delta(w, R)}|f|^{2} d \mu \leq e^{3 R^{2}} \int_{\Delta(w, 2 R) \backslash \Delta(w, R)}|f|^{2} d \mu .
$$

Adding $\int_{C \backslash \Delta(w, R)}|f|^{2} d \mu$ to both sides of (2.2) implies the assertion with $X=$ $\Delta(w, R)$ and $C:=e^{3 R^{2}}+1$.

We now proceed with the multi-dimensional case. Assume that $X$ satisfies the assumptions of the lemma with some $k$. Let $R$ be the constant defined in the statement of Lemma, then $P_{k}(X) \subseteq \Delta(w, R+1)$ with some $w \in \mathrm{C}$. Let $f: \mathrm{C}^{n} \rightarrow \mathrm{C}$ be an entire function. Set $Y_{k}:=\left\{\left(z_{1}, \ldots, z_{n}\right) \in \mathrm{C}^{n}:\left|z_{k}-w\right|>\right.$ $R+1\}$. An easy application of the one-dimensional case leads to

$$
\int_{C^{n}}|f|^{2} d \mu \leq C \int_{Y_{k}}|f|^{2} d \mu
$$

with $C:=e^{3(R+1)^{2}}+1$. Since $Y_{k} \subseteq \mathrm{C}^{n} \backslash X$ the result follows.

Lemma 2.2. Given $f \in \mathscr{B}_{1}, p \in \mathscr{P}_{1} \backslash\{0\}$ and $\epsilon>0$ there exists a polynomial $q \in \mathscr{P}_{1}$ such that $\|f-q\| \leq \epsilon$ and $f=q(\bmod p)$, i.e. there exists $h \in \operatorname{Hol}(\mathrm{C})$ satisfying $f-q=p h$.

Proof. We first state the following auxiliary fact:

Lemma 2.3. Let $p \in \mathscr{P}_{1} \backslash\{0\}$ and let $\rho>0$ be such that $\Delta(0, \rho)$ contains all zeroes of $p$. Then there exists a constant $C>0$ depending only on $p$ and $\rho$ such that for every $F \in \operatorname{Hol}(\Delta(0, \rho))$ one can find a unique $Q \in \mathscr{P}_{1}$ such that

(i) $\operatorname{deg} Q \leq \operatorname{deg} p-1$,

(ii) $\frac{F-Q}{p} \in \operatorname{Hol}(\Delta(0, \rho))$,

(iii) $\sup _{z \in \Delta(0, \rho)}|Q(z)| \leq C \sup _{z \in \Delta(0, \rho)}|F(z)|$.

To prove this suppose that $z_{1}, \ldots, z_{r}$ are distinct zeroes of $p$ with multiplicities $m_{1}, \ldots, m_{r}$ (resp.). Observe that $m:=m_{1}+\ldots m_{r}=\operatorname{deg} p$. By the Cauchy inequality there exists $C_{1}>0$ (depending on $\rho$ ) such that

$$
\left|g^{(l)}\left(z_{j}\right)\right| \leq C_{1} \sup _{z \in \Delta(0, \rho)}|g(z)|, \quad l=1, \ldots, m_{j},
$$

for every $j \in\{1, \ldots, r\}$ and $g \in \operatorname{Hol}(\Delta(0, \rho))$. We can find another constant $C_{2}>0$, which depends only on $p$ and $\rho$, such that

$$
\sup _{z \in \Delta(0, \rho)}|h(z)| \leq C_{2} \max \left\{\left|h^{\left(l_{j}\right)}\left(z_{j}\right)\right|: l_{j}=1, \ldots, m_{j} \text { and } j=1, \ldots, r\right\},
$$


for every $h \in \mathscr{P}_{1}$ with $\operatorname{deg} h \leq m-1$, because by the Lagrange-Hermite interpolation the right-hand side of the above inequality defines a norm in the space of polynomials of degree at most $m-1$.

Choose arbitrary $F \in \operatorname{Hol}(\Delta(0, \rho))$. Applying the Lagrange-Hermite interpolation we infer that there exists a unique $Q \in \mathscr{P}_{1}$ of degree at most $m-1$ such that

$$
Q^{(l)}\left(z_{j}\right)=F^{(l)}\left(z_{j}\right), \quad l=1, \ldots, m_{j} .
$$

for every $j=1, \ldots, r$. Hence $Q$ satisfies (i) and (ii). By the choice of $C_{1}$ and $C_{2}$ we see that (iii) holds with $C:=C_{1} C_{2}$. This completes the proof of Lemma 2.3.

We now turn to the proof of Lemma 2.2. Fix $f \in \mathscr{B}_{1}, p \in \mathscr{P}_{1}$ and $\delta>0$. Choose a polynomial $g$ such that $\|f-g\| \leq \delta$ and $\rho>0$ such that $\Delta(0, \rho)$ contains all zeroes of $p$. Set $F:=f-g$. Then, by the reproducing property for $\mathscr{B},|F(z)| \leq \delta e^{|z|^{2} / 2}, z \in \mathrm{C}$, and, consequently,

$$
|F(z)| \leq \delta e^{\rho^{2} / 2}, \quad z \in \Delta(0, \rho) .
$$

We can now apply Lemma 2.3 to obtain the polynomial $Q$, deg $Q \leq m-1$ (here $m:=\operatorname{deg} p$ ), such that $\frac{F-Q}{p}$ is analytic in $\Delta(0, \rho)$ (hence entire) and

$$
|Q(z)| \leq C \delta e^{\rho^{2} / 2}, \quad z \in \Delta(0, \rho),
$$

with $C>0$ depending only on $p$ and $\rho$. This implies that

$$
|Q(z)| \leq C \delta e^{\rho^{2} / 2} \max \left\{\left(\frac{|z|}{\rho}\right)^{m-1}, 1\right\}, \quad z \in \mathrm{C} .
$$

This inequality leads to $\|Q\| \leq C^{\prime} \delta$ with a new constant $C^{\prime}>0$ depending only on $p$ and $\rho$. Setting $q:=g+Q$ we obtain a polynomial, for which $f-q$ is divisible by $p$. Moreover,

$$
\|f-q\|=\|f-g-Q\| \leq\|f-g\|+\|Q\| \leq \delta\left(1+C^{\prime}\right) .
$$

Since $\delta$ can be made arbitrarily small, the result follows.

Theorem 2.4. Let $p \in \mathscr{P}_{1} \otimes B\left(\mathrm{C}^{M}, \mathrm{C}^{N}\right)$ with $M>N \geq 1$ and $f \in \mathscr{D}\left(T_{p}\right)$. Assume that $p(z)$ is of maximal rank for at least one point $z \in C$. Suppose that $T_{p} f=0$. Then for any $\epsilon>0$ there exists $q \in \mathscr{P}_{1} \otimes \mathrm{C}^{M}$ such that $\|f-q\|_{(M)} \leq \epsilon$ and $T_{p} q=0$.

Proof. We can represent $p$ by means of the matrix $\left[p_{k l}\right]_{k=1, \ldots, N}^{l=1, \ldots, M}$, where $p_{k l} \in \mathscr{P}_{1}$. Put $\tilde{p}:=\left[p_{k l}\right]_{k=1, \ldots, N}^{l=1, \ldots, N}$. By assumption on the rank of $p$, rearranging columns if necessary, we can assume that $d(z):=\operatorname{det} \tilde{p}(z)$ is not identically 
zero; moreover, it can be done so that deg $d$ is maximal among degrees of all possible minors of dimension $N$, which arise from the matrix representing $p$.

Given $\epsilon_{0}>0$ by Lemma 2.2 we can find polynomials $q_{N+1}, \ldots, q_{M} \in$ $\mathscr{P}_{1}$ such that $f_{j}=q_{j}(\bmod d)$ and $\left\|f_{j}-q_{j}\right\|<\epsilon_{0}, j=N+1, \ldots, M$. By the Cramer formulas there exist rational functions $q_{1}, \ldots, q_{N}$ such that $T_{p}\left(q_{1}, \ldots, q_{M}\right)=0$. Thus every $q_{j}, j=1, \ldots, N$, can be expressed by $q_{N+1}, \ldots, q_{M}$ via

$$
q_{j}=d^{-1} \operatorname{det}\left[\begin{array}{ccccccc}
p_{11} & \ldots & p_{1 j-1} & -\sum_{l=N+1}^{M} p_{1 l} q_{l} & p_{1 j+1} & \ldots & p_{1 N} \\
\vdots & & \vdots & \vdots & \vdots & & \vdots \\
p_{N 1} & \ldots & p_{N j-1} & -\sum_{l=N+1}^{M} p_{N l} q_{l} & p_{N j+1} & \ldots & p_{N N}
\end{array}\right]
$$

for $j=1, \ldots, N$.

Observe that all $q_{j}$ 's are polynomials! Indeed, one can verify that the difference $f_{j}-q_{j}$ is an entire function. To see this it suffices to check that the formula (2.3) holds if $q_{j}$ is replaced by $f_{j}, j=1, \ldots, M$, in both members of (2.3). Then it turns out that for each $j \in\{1, \ldots, N\}$ function $f_{j}-q_{j}$ is a linear combination with polynomial coefficients of $f_{l}-q_{l}, l=N+1, \ldots, M$, divided by $d$. By the choice of $q_{j}$ the difference $f_{l}-q_{l}$ is divisible by $d, l=N+1, \ldots, M$. Thus $f_{j}-q_{j}$ is entire and, consequently, $q_{j}$ is entire. Since every entire rational function has to be a polynomial, we deduce that every $q_{j}$ is a polynomial.

It remains to estimate the norm of $f_{j}-q_{j}$ for $j=1, \ldots, N$. Note that by (2.3) for fixed $j \in\{1, \ldots, N\}$ we get

$$
f_{j}-q_{j}=\sum_{l=N+1}^{M} \alpha_{l} \frac{M_{l}}{d}\left(f_{l}-q_{l}\right),
$$

where $M_{l}$ is a properly chosen minor of dimension $N$ in $p$ (obviously, $M_{l}$ depends on $j$, which has been omitted in the notation) and $\alpha_{l}$ is equal to 1 or -1 . Choose $R>0$ such that all zeros of $d$ lie in $\Delta_{R}(=\Delta(0, R))$. By the assumption on $\operatorname{deg} d$ we infer that every quotient $\frac{M_{l}}{d}$ is bounded outside $\Delta_{R}$. Set $\tilde{c}_{l}=\sup \left\{\left|\frac{M_{l}(z)}{d(z)}\right|: z \in \mathrm{C} \backslash \Delta_{R}\right\}$. Then

$$
\left|f_{j}(z)-q_{j}(z)\right| \leq \sum_{l=N+1}^{M} \tilde{c}_{l}\left|f_{l}(z)-q_{l}(z)\right|, \quad z \in \mathrm{C} \backslash \Delta_{R} .
$$

Taking squares, integrating over $C \backslash \Delta_{R}$ and applying Lemma 2.1 yield

$$
\left\|f_{j}-q_{j}\right\|^{2} \leq C_{R} \epsilon_{0}^{2}(M-N) \sum_{l=N+1}^{M} \tilde{c}_{l}^{2},
$$


where $C_{R}$ is some positive constant. If $\epsilon_{0}$ is small enough, then polynomial $q:=\left(q_{1}, \ldots, q_{M}\right)$ satisfies all the required conditions.

NotE. The authors thank John McCarthy for a valuable suggestion in connection with the proof of Theorem 2.4.

Theorem 2.5. Let $p \in \mathscr{P}_{1} \otimes B\left(\mathrm{C}^{M}, \mathrm{C}^{N}\right)$. Then $T_{p}^{*}=\Pi_{p^{*}}$.

Proof. It suffices to check the density of $\mathscr{P}_{1} \otimes \mathrm{C}^{M}$ in $\mathscr{D}\left(T_{p}\right)$ with respect to the graph norm $\|\cdot\|_{T_{p}}$. Choose $f \in \mathscr{D}\left(T_{p}\right)$. We are going to show that there exists $q \in \mathscr{P}_{1} \otimes \mathrm{C}^{M}$ such that $\|f-q\|_{T_{p}}$ is arbitrarily small. Define $\tilde{p} \in \mathscr{P}_{1} \otimes B\left(\mathrm{C}^{M+N}, \mathrm{C}^{N}\right)$ by $\tilde{p}(z)=\left[p(z),-I_{N}\right]$, where $I_{N}$ stands for the identity matrix of dimension $N$. It is easily seen that $\tilde{p}$ satisfies the assumptions of Theorem 2.4. Since $T_{\tilde{p}}\left(f, T_{p} f\right)=0$, given $\epsilon>0$ we can find $\tilde{q}=$ $\left(q_{1}, \ldots, q_{M+N}\right) \in \mathscr{P}_{1} \otimes \mathrm{C}^{M+N}$ such that $T_{\tilde{p}} \tilde{q}=0$ and $\left\|\left(f, T_{p} f\right)-\tilde{q}\right\|_{(M+N)} \leq$ $\epsilon$. Setting $q:=\left(q_{1}, \ldots, q_{N}\right)$ we see that $T_{p} q=\left(q_{N+1}, \ldots, q_{N+M}\right)$, as a consequence of the equality $T_{\tilde{p}} \tilde{q}=0$, and $\|f-q\|_{T_{p}}^{2}=\left\|\left(f, T_{p} f\right)-\tilde{q}\right\|_{(M+N)}^{2} \leq \epsilon^{2}$.

It seems that the case of homogeneous polynomials is much easier to deal with, even in the case of several complex variables and values being operators on (possibly) infinite-dimensional Hilbert space $\mathscr{H}$.

Proposition 2.6. If $p \in \mathscr{P} \otimes B(\mathscr{H})$ is a homogeneous polynomial then $T_{p}^{*}=\Pi_{p^{*}}$

Proof. The main idea of the proof is to write $\mathscr{B} \otimes \mathscr{H}$ as an orthogonal sum of subspaces reducing $p^{\#}(D) T_{p}$ to a non-negative operator. Let $\mathscr{F}_{k} \subseteq \mathscr{P} \otimes \mathscr{H}$ denote the space of homogeneous polynomials of degree $k$ (with the zero polynomial included). Obviously, $\mathscr{B} \otimes \mathscr{H}=\bigoplus_{k=0}^{\infty} \mathscr{F}_{k}$. It is easily seen that $p^{\#}(D) T_{p}\left(\mathscr{F}_{k}\right) \subseteq \mathscr{F}_{k}$ for all $k \geq 0$. Hence, each $\mathscr{F}_{k}$ reduces $p^{\#}(D) T_{p}$. Pick arbitrary $f \in \mathscr{F}_{k}$ and compute

$$
\begin{aligned}
\left\langle p^{\#}(D) T_{p} f, f\right\rangle_{(\mathscr{H})} & =\int_{C^{n}}\left\langle p^{\#}(D)(p f)(z), f(z)\right\rangle_{\mathscr{C}} d \mu(z) \\
& =\int_{C^{n}} \int_{C^{n}}\left\langle p(\zeta)^{*} p(\zeta) f(\zeta), f(z)\right\rangle_{\mathscr{H}} e^{z \cdot \bar{\zeta}} d \mu(\zeta) d \mu(z) .
\end{aligned}
$$

The last equality follows from [4, Lemma 2.3], but the reader may obtain it applying the theorem on differentiating under the integral sign. We may now change the order of integration, which is allowed because the function under the integral sign is summable with respect to $\mu \otimes \mu$. Then it suffices to apply the reproducing property for $\mathscr{B} \otimes \mathscr{H}$ to see that

$$
\left\langle p^{\#}(D) T_{p} f, f\right\rangle_{(\mathscr{H})}=\left\langle p^{*} p f, f\right\rangle_{(\mathscr{H})} \geq 0 .
$$


Since $^{3} p^{\#}(D) T_{p}=\left.\bigoplus_{k=0}^{\infty} p^{\#}(D) T_{p}\right|_{\mathscr{F}_{k}}$ we infer that $p^{\#}(D) T_{p}$ is non-negative, so by Lemma 1.1 the assertion follows.

REMARK 2.7. The above prove works also for the wider class of $t$-homogeneous polynomials (cf. [9]).

Proposition 2.8. Assume that $p=\left[p_{k l}\right]_{k, l=1, \ldots, N} \in \mathscr{P} \otimes B\left(C^{N}\right)$ is such that all $p_{k l}$ are homogeneous. If $\operatorname{deg} p_{k l}$ depends only on l, then $T_{p}^{*}=\Pi_{p^{*}}$.

Proof. (Based on an idea of J. Janas.) We are going to prove that $T_{p} p^{\#}(D)$ is non-negative. Let $\mathscr{F}_{k, j} \subseteq \mathscr{P} \otimes \mathrm{C}^{j}$ denote the space of homogeneous polynomials of degree $k$ for $k \geq 0$. Observe that $\mathscr{B} \otimes \mathrm{C}^{N}=\bigoplus_{k=0}^{\infty} \mathscr{F}_{k, N}$. We claim that each $\mathscr{F}_{k, N}$ is invariant under $T_{p} p^{\#}(D)$ (hence reducing for this operator). Operator $\left.T_{p} p^{\#}(D)\right|_{\mathscr{F}_{k, N}}: \mathscr{F}_{k, N} \rightarrow \mathscr{B} \otimes \mathrm{C}^{N}$ may be written as an operator matrix $\left[A_{k l}\right]_{k, l=1, \ldots, N}$, where

$$
A_{k l}=\sum_{j=0}^{N} T_{p_{k j}} p_{l j}^{\#}(D), \quad \mathscr{D}\left(A_{k l}\right)=\mathscr{F}_{k, 1} .
$$

By assumption on $p$ we see that if $f$ is homogeneous of degree $k$ then so is $A_{k l} f$.

It now remains to show that $\left.T_{p} p^{\#}(D)\right|_{\mathscr{F}_{k, N}}$ is non-negative. Note first that

$$
\begin{aligned}
P \otimes I_{\mathrm{C}^{N}}\left(p^{*} f\right)(z) & =\int_{\mathrm{C}^{n}} p^{*}(\zeta) f(\zeta) e^{z \cdot \bar{\zeta}} d \mu(\zeta), \quad z \in \mathrm{C}^{n}, \quad f \in \mathscr{F}_{k, N}, \\
& =p^{\#}(D) f(z)
\end{aligned}
$$

for all $k \geq 0$ (cf. [4, Lemma 2.3]). Thus

$$
\left\langle T_{p} p^{\#}(D) f, f\right\rangle_{(\mathscr{H})}=\left\langle p p^{\#}(D) f, f\right\rangle_{(\mathscr{H})}=\left\langle p^{\#}(D) f, P \otimes I_{\mathrm{C}^{N}}\left(p^{*} f\right)\right\rangle_{(\mathscr{H})} \geq 0
$$

for all $f \in \mathscr{F}_{k, N}$ and $k \geq 0$. Applying Lemma 1.1 completes the proof.

One of the natural questions which arise when studying the adjointness hypothesis is whether it is possible to find the solution to this problem only by means of coefficients of $p$. This idea is presented in the following theorem. The set of operators $\mathscr{A} \subseteq B(\mathscr{H})$ is called jointly subnormal if there exists a set $\mathscr{A}^{\prime} \subseteq B(\mathscr{K})$ of commuting normal operators defined on a larger Hilbert space containing $\mathscr{H}$ as a closed subspace such that for every $A \in \mathscr{A}$ there exists $A^{\prime} \in \mathscr{A}^{\prime}$ satisfying $A=\left.A^{\prime}\right|_{\mathscr{H}}$.

\footnotetext{
${ }^{3}$ Note that $p^{\#}(D) T_{p}$ is closed.
} 
TheOREM 2.9. Suppose that $p=\sum_{k=0}^{N} p_{k} \otimes A_{k} \in \mathscr{P} \otimes B(\mathscr{H})$ with $p_{k} \in \mathscr{P}$ and $A_{k} \in B(\mathscr{H})$. If the sequence $\left\{A_{k}\right\}_{k=0}^{N}$ is jointly subnormal, then $T_{p}^{*}=\Pi_{p^{*}}$.

The proof of this theorem requires the following lemma

Lemma 2.10. Suppose that $q \in \mathscr{P} \otimes B(\mathscr{H})$ takes values only among normal operators, $f \in \mathscr{D}\left(T_{q}\right)$ and $g \in \mathscr{B} \otimes \mathscr{H}$. Then the function

$$
\mathrm{C}^{n} \times \mathrm{C}^{n} \ni(z, w) \rightarrow\langle g(w), q(w) f(z)\rangle e^{z \cdot \bar{w}} \in \mathrm{C}
$$

is summable with respect to the measure $\mu \otimes \mu$ in $\mathrm{C}^{n} \times \mathrm{C}^{n}$.

Proof. We begin with a change of variables $w=z+\zeta$, which yields

$$
\int_{C^{n}}\left|\langle g(w), q(w) f(z)\rangle e^{z \cdot \bar{w}}\right| d \mu(w)=\int_{C^{n}}\left|\langle g(z+\zeta), q(z+\zeta) f(z)\rangle e^{-z \cdot \bar{\zeta}}\right| d \mu(\zeta)
$$

Thus

$$
\begin{aligned}
J & :=\int_{C^{n}} \int_{C^{n}}\left|\langle g(w), q(w) f(z)\rangle e^{z \cdot \bar{w}}\right| d \mu(w) d \mu(z) \\
& \leq \int_{C^{n}}\left\|g(\cdot+\zeta) e_{-\zeta}\right\|_{(\mathscr{H})}\|q(\cdot+\zeta) f\|_{(\mathscr{H})} d \mu(\zeta) .
\end{aligned}
$$

But $\left\|g(\cdot+\zeta) e_{-\zeta}\right\|_{(\mathscr{C})}=e^{\frac{1}{2}\|\zeta\|^{2}}\|g\|_{(\mathscr{H})}$, which together with the normality of $q(z)$ for all $z \in \mathrm{C}^{n}$ implies that

$$
J \leq\|g\|_{(\mathscr{H})} \int_{\mathrm{C}^{n}}\left\|q(\cdot+\zeta)^{*} f\right\|_{(\mathscr{H})} e^{\frac{1}{2}\|\zeta\|^{2}} d \mu(\zeta) .
$$

Applying the isometry theorem (cf. [10], [4]), which states that ${ }^{4}$

$$
\left\|p^{*} h\right\|_{(\mathscr{H})}^{2}=\sum_{j \geq 0} \frac{1}{j !}\left\|\left(D^{j} p^{\#}\right)(D) h\right\|_{(\mathscr{H})}^{2}, \quad p \in \mathscr{P} \otimes B(\mathscr{H}), \quad h \in \mathscr{B} \otimes \mathscr{H},
$$

we infer that $\left\|q(\cdot+\zeta)^{*} f\right\|_{(\mathscr{C})}$ is of polynomial growth, so $J<\infty$.

Proof of Theorem 2.9. Let $q(z):=\sum_{j=0}^{N} p_{j} \otimes B_{j}$, where $\left\{B_{k}\right\}_{k=0}^{N}$ is the sequence of commuting normal operators defined on a larger Hilbert space $\mathscr{K}$ such that $A_{k}=\left.B_{k}\right|_{\mathscr{H}}, k=0, \ldots, N$. Thus $q$ takes its values only among normal operators and, consequently, $T_{q}^{*}=q^{\#}(D)$, according to $[4$, Theorem

\footnotetext{
${ }^{4}$ The symbol " $\sum_{j \geq 0}$ " should be read "sum over all multiindices $j$ ". In the following equality we put $\|F\|_{(\mathscr{H})}=\infty$ whenever $F \notin L^{2}(\mu) \otimes \mathscr{H}$
} 
7.8]. We will show that $\left\langle p^{\#}(D) T_{p} f, f\right\rangle_{(\mathscr{C})} \geq 0$ for every $f \in \mathscr{D}\left(p^{\#}(D) T_{p}\right)$. Observe that

$$
\begin{aligned}
\left\langle p^{\#}(D) T_{p} f, f\right\rangle_{(\mathscr{H})} & =\int_{C^{n}}\left\langle p^{\#}(D)(p f)(z), f(z)\right\rangle d \mu(z) \\
& =\int_{C^{n}}\left\langle q^{\#}(D)(p f)(z), f(z)\right\rangle d \mu(z) \\
& =\int_{C^{n}} \int_{C^{n}}\left\langle q(w)^{*} p(w) f(w), f(z)\right\rangle e^{z \cdot \bar{w}} d \mu(w) d \mu(z)
\end{aligned}
$$

We know that $p f \in \mathscr{B} \otimes \mathscr{H}$, which implies that $q f \in \mathscr{B} \otimes \mathscr{K}$, because $p(z)=\left.q(z)\right|_{\mathscr{H}}$ for all $z \in \mathrm{C}^{n}$. By Lemma 2.10 we are allowed to change the order of integration in (2.4). So we arrive at

$$
\begin{aligned}
\left\langle p^{\#}(D) T_{p} f, f\right\rangle_{(\mathscr{H})} & =\int_{C^{n}} \int_{C^{n}}\left\langle q(w)^{*} p(w) f(w), f(z)\right\rangle e^{z \cdot \bar{w}} d \mu(z) d \mu(w) \\
& =\int_{C^{n}}\left\langle q(w)^{*} p(w) f(w), f(w)\right\rangle d \mu(w) \\
& =\int_{C^{n}}\|p(w) f(w)\|^{2} d \mu(w) \geq 0 .
\end{aligned}
$$

Thus $\operatorname{ker}\left(I_{\mathscr{C}}+p^{\#}(D) T_{p}\right)=\{0\}$, which implies that $T_{p}^{*}=p^{\#}(D)$.

REMARK 2.11. If the property $T_{p}^{*}=\Pi_{p^{*}}$ is proved for all polynomials, whose coefficients commute, then it can easily be shown for arbitrary polynomial $p$. To see this pick arbitrary $p \in \mathscr{P} \otimes B(\mathscr{H})$ and define polynomial $q \in \mathscr{P} \otimes B(\mathscr{H} \oplus \mathscr{H})$ via

$$
q(z)=\left[\begin{array}{cc}
0 & 0 \\
p(z) & 0
\end{array}\right], \quad z \in \mathrm{C}^{n} .
$$

Note that $q(z)$ can be written as $\sum_{|j| \leq N} B_{j} z^{j}, z \in \mathrm{C}^{n}$, where $B_{j} \in B(\mathscr{H} \oplus \mathscr{H})$ and $B_{j} B_{k}=0$ for all admissible $j$ and $k$. Suppose that $T_{q}=q^{\#}(D)$. Then for any $g \in \mathscr{D}\left(T_{q}\right)$ one can find a sequence $\left\{h_{j}\right\}_{j=0}^{\infty}$ in $\mathscr{P} \otimes(\mathscr{H} \oplus \mathscr{H})$ such that $h_{j} \rightarrow g$ and $q h_{j} \rightarrow q g$. One can readily check that $\|q(z)(f \oplus g)\|=\|p(z) f\|$ for all $z \in \mathrm{C}^{n}$ and $f, g \in \mathscr{H}$.

Pick arbitrary $f \in \mathscr{D}\left(T_{p}\right)$. Then $f \oplus 0$ belongs to the domain of $T_{q}$. Let $\left\{h_{j}\right\}_{j=0}^{\infty} \subseteq \mathscr{P} \otimes(\mathscr{H} \oplus \mathscr{H})$ be the sequence chosen so that $h_{j} \rightarrow f \oplus 0$ and $q h_{j} \rightarrow q(f \oplus 0)$. Let $P_{\mathscr{L} \oplus 0}$ be the orthogonal projection of $\mathscr{H} \oplus \mathscr{H}$ onto $\mathscr{H} \oplus 0$. By the choice of $q$ it is apparent that the sequence $\left\{P_{\mathscr{C} \oplus 0} h_{j}\right\}_{j=0}^{\infty} \subseteq \mathscr{P} \otimes \mathscr{H}$ tends 
to $f$ in the graph norm of $T_{p}$. Thus $\mathscr{P} \otimes \mathscr{H}$ is a core for $T_{p}$ and, consequently, $T_{p}^{*}=p^{\#}(D)$.

\section{Closedness of range}

Let $p \in \mathscr{P} \otimes B\left(\mathrm{C}^{N}\right)$. In what follows, det $p$ stands for the polynomial defined in the natural way as $(\operatorname{det} p)(z)=\operatorname{det}(p(z))$, and $p^{k l} \in B\left(\mathrm{C}^{N-1}\right)$ originates from $p$ by removing the $k$-th row and the $l$-th column.

Theorem 3.1. Assume that $p \in \mathscr{P}_{1} \otimes B\left(\mathrm{C}^{N}\right), p \neq 0$. Then $T_{p}$ is bounded below if and only if $\operatorname{deg} \operatorname{det} p \geq \max _{k, l=1, \ldots, N} \operatorname{deg} \operatorname{det} p^{k l} \geq 0$.

Proof. The "only if" part of assertion was proved in [4, Proposition 8.6]. It remains to consider the "if" part. Suppose that $p=\left[p_{k l}\right]_{k, l=1, \ldots, N}$ with $p_{k l} \in \mathscr{P}_{1}$ and $T_{p}\left(f_{1}, \ldots, f_{N}\right)=\left(g_{1}, \ldots, g_{N}\right)$ for $\left(f_{1}, \ldots, f_{N}\right) \in \mathscr{D}\left(T_{p}\right)$, which means that

$$
\left[\begin{array}{ccc}
p_{11} & \ldots & p_{1 N} \\
\vdots & & \vdots \\
p_{N 1} & \ldots & p_{N N}
\end{array}\right]\left[\begin{array}{c}
f_{1} \\
\vdots \\
f_{N}
\end{array}\right]=\left[\begin{array}{c}
g_{1} \\
\vdots \\
g_{N}
\end{array}\right] .
$$

Note that by assumption det $p$ is not identically 0 , so the above equation may be solved for $f_{1}, \ldots, f_{N}$. Choose $R>0$ such that all zeroes of det $p$ lie in $\Delta(0, R)$. Hence, by the Cramer formulas, we infer that

$$
\left|f_{j}(z)\right| \leq \sup \left\{\left|\frac{\operatorname{det} p^{k l}(\zeta)}{\operatorname{det} p(\zeta)}\right|: k, l=1, \ldots, N,|\zeta| \geq R\right\}\left(\left|g_{1}(z)\right|+\ldots\left|g_{N}(z)\right|\right)
$$

for all $j=1, \ldots N$ and $z \in C \backslash \Delta(0, R)$. Let $M$ denote the supremum in the above inequality. Then it follows that

$$
\int_{C \backslash \Delta(0, R)} \sum_{j=1}^{N}\left|f_{j}\right|^{2} d \mu \leq M N \sum_{j=1}^{N}\left\|g_{j}\right\|^{2} .
$$

Applying Lemma 2.1 yields

$$
\left\|T_{p}\left(f_{1}, \ldots, f_{N}\right)\right\|_{(N)}=\left\|\left(g_{1}, \ldots, g_{N}\right)\right\|_{(N)} \geq C\left\|\left(f_{1}, \ldots, f_{N}\right)\right\|_{(N)}
$$

with an appropriate constant $C>0$ depending only on $p$ and $R$. This means that $T_{p}$ is bounded below.

Theorem 3.1 is the one variable refinement of [4, Proposition 8.6], which gives the necessary condition for boundedness below of $T_{p}$ in the multivariable case. Although it does not seem likely one can obtain the multivariable version of the above theorem we have not been able to give any counterexample. 
The following proposition deals with non-injective operators with closed range.

Proposition 3.2. Let $p \in \mathscr{P}_{1} \otimes B\left(\mathrm{C}^{N}, \mathrm{C}\right)$. Then $\mathscr{R}\left(T_{p}\right)$ is closed.

Proof. Assume that $p=\left[p_{1}, \ldots, p_{N}\right]$ and $q$ is the greatest common divisor of all $p_{j}$ 's, $j=1, \ldots, N$. We may choose $p$ such that $p_{N} \neq 0$, which involves no loss of generality. We are going to establish the following description of the range:

$$
\mathscr{R}\left(T_{p}\right)=\left\{g \in \mathscr{B}_{1}: \frac{g}{q} \text { extends to an entire function }\right\} .
$$

Since $T_{p}\left(f_{1}, \ldots, f_{N}\right)=\sum_{j=1}^{N} p_{j} f_{j}$ the inclusion " $\subseteq$ " is obvious. To prove the reverse inclusion pick $g \in \mathscr{B}_{1}$ such that $\frac{g}{q}$ is entire. By easy algebra there exist polynomials $q_{1}, \ldots, q_{j}$ such that $\sum_{j=1}^{N} q_{j} p_{j}=q r$, where $r$ is a polynomial chosen so that $\frac{g-q r}{p_{N}}$ is entire (use the interpolation property). Put

$$
f_{j}:=q_{j} \quad \text { for } j=1, \ldots, N-1, \quad \text { and } \quad f_{N}:=\frac{g-q r}{p_{N}}+q_{N} .
$$

It is easily seen that $f_{N} \in \mathscr{B}_{1}$ and $T_{p}\left(f_{1}, \ldots, f_{N}\right)=g$, thus $g \in \mathscr{R}\left(T_{p}\right)$.

Observe that $\mathscr{R}\left(T_{p}\right)=\mathscr{R}\left(T_{q}\right)$, where $T_{q}$ is Toeplitz operator defined in $\mathscr{B}_{1}$. By the Newman-Shapiro Isometry Theorem [10], [4] operator $T_{q}$ is bounded below, hence $\mathscr{R}\left(T_{q}\right)$ is closed.

We now turn to an example showing that the above proposition is not true in the multivariable case.

Example 3.3. $\mathscr{R}\left(T_{p}\right)$ need not be closed even in case of homogeneous polynomials. Define $p=\left[\begin{array}{ll}p_{1} & p_{2}\end{array}\right]$, where $p_{1}(z, w, \zeta)=z^{3} w^{3}-\zeta^{6}$ and $p_{2}(z, w, \zeta)=w^{6},(z, w, \zeta) \in \mathrm{C}^{3}$. Observe that $\mathscr{R}\left(T_{p}\right)$ contains all functions of type $w^{3} \zeta^{6} q$, where $q$ is an arbitrary polynomial depending only on $z$. This follows from $T_{p}\left(-w^{3} q, z^{3} q\right)=w^{3} \zeta^{6} q$. Suppose $\mathscr{R}\left(T_{p}\right)$ is closed. Then it follows that the closure of $\left\{w^{3} \zeta^{6} q: q\right.$ is a polynomial depending only on $\left.z\right\}$ is contained in $\mathscr{R}\left(T_{p}\right)$. But this closure is equal to $\left\{w^{3} \zeta^{6} f: f \in \mathscr{B}_{3}, f\right.$ depends only on $z\}$, which is an immediate consequence of $\left\|w^{3} \zeta^{6} q\right\|^{2}=3 ! 6 !\|q\|^{2}$, with $q$ depending only on $z$. Put $f_{0}(z)=\left(e^{z^{2} / 2}-1\right)\left(z-z_{1}\right)^{-1}\left(z-z_{2}\right)^{-1}$, where $z_{1}, z_{2}$ are two different zeroes of function $e^{z^{2} / 2}-1$. Observe that $f_{0} \in \mathscr{B}_{1}$. Hence, there are $f, g \in \mathscr{B}_{3}$ such that $T_{p}(f, g)(z, w, \zeta)=w^{3} \zeta^{6} f_{0}(z)$, or more explicitly

$$
\left(z^{3} w^{3}-\zeta^{6}\right) f(z, w, \zeta)+w^{6} g(z, w, \zeta)=w^{3} \zeta^{6} \frac{e^{z^{2} / 2}-1}{\left(z-z_{1}\right)\left(z-z_{2}\right)}, z, w, \zeta \in \mathbf{C} .
$$


Fix arbitrary $x>0$. Substituting $z:=x, w:=\frac{1}{x}$ and $\zeta:=1$ we get

$$
g\left(x, \frac{1}{x}, 1\right)=x^{3} \frac{e^{x^{2} / 2}-1}{\left(x-z_{1}\right)\left(x-z_{2}\right)}, \quad x>0 .
$$

This leads to a contradiction, because the left hand side of the above equality can be estimated from above by $\|g\| \exp \left(\frac{x^{2}}{2}+\frac{1}{2 x^{2}}+\frac{1}{2}\right), x>0$, whereas this is impossible for the right hand side. So we have proved that the range of $T_{p}$ is not closed.

By a similar argument one can show that the range of operator $T_{q}$ with $q(z, w, \zeta)=\left[q_{1} q_{2}\right], q_{1}(z, w, \zeta)=z^{3} w^{3}-\zeta^{6}, q_{2}(z, w, \zeta)=w^{4},(z, w, \zeta) \in$ $\mathrm{C}^{3}$, is not closed. Observe that $\operatorname{deg} q_{1} \neq \operatorname{deg} q_{2}$ in opposition to the previous example. Hence, if we drop the assumption on equality of degrees of coordinate polynomials, then it does not follow that the range of $T_{p}$ is closed in case of several complex variables.

\section{The non-density example}

Below we show an example of an analytic function $\varphi$ for which polynomials do not form a core for the operator $T_{\varphi}$ though they are contained in its domain. In other words: we will indicate an analytic $\varphi$ for which the space of all entire functions square-integrable with respect to the measure $\left(1+|\varphi|^{2}\right) d \mu$ contains polynomials as a non-dense subset. Before we go into details we need the following theorem, which is of independent interest.

THEOREM 4.1. Let $\left\{z_{k}\right\}_{k=1}^{\infty} \subseteq \mathrm{C}$ be a sequence of non-zero numbers satisfying $\left|\frac{z_{k+1}}{z_{k}}\right| \geq \lambda$ for all $k \in \mathrm{N}$ with some $\lambda>1$. Then the formula

$$
\psi(z):=\prod_{k=1}^{\infty}\left(1-\frac{z}{z_{k}}\right), \quad z \in \mathrm{C},
$$

defines an entire function for which there exists $c>0$ such that $\left\|T_{\psi} f\right\| \geq c\|f\|$ for all $f \in \mathscr{D}\left(T_{\psi}\right)$.

Proof. Since we want to impose some additional conditions on the given sequence $\left\{z_{k}\right\}_{k=1}^{\infty} \subseteq \mathrm{C}$, we will show that it suffices to deal only with a modification of this sequence obtained by removing a few initial terms. Indeed, if the theorem is proved for $\psi_{1}(z):=\prod_{k=1}^{\infty}\left(1-\frac{z}{z_{k+j}}\right)$ with some integer $j \geq 0$, then applying the boundedness below of $T_{p}$ with the polynomial $p:=\frac{\psi}{\psi_{1}}$ we get

$$
\left\|T_{\psi} f\right\|=\left\|T_{p} \psi_{1} f\right\| \geq c_{1}\left\|\psi_{1} f\right\|, \quad f \in \mathscr{D}\left(T_{\psi}\right),
$$

where $c_{1}$ is a positive constant. Thus if $T_{\psi_{1}}$ is bounded below, then so is $T_{\psi}$. 
From now on we are going to assume that $\left|z_{1}\right| \geq 1$,

$$
\lambda \geq \frac{3}{\left|z_{k}\right|}+1, \quad k=1,2, \ldots,
$$

and

$$
\left|z_{k}-z_{j}\right|>4, \quad k \neq j,
$$

which can be easily obtained by omitting a suitable number of initial terms in the sequence $\left\{z_{k}\right\}_{k=1}^{\infty}$.

Denote by $\Delta(w, r)$ the open disk with radius $r$ centered at $w \in \mathrm{C}$. We claim that there exists a positive constant $a$ such that

$$
|\psi(z)| \geq a \quad \text { for } \quad z \in \mathrm{C} \backslash \bigcup_{k=1}^{\infty} \Delta\left(z_{k}, 1\right) .
$$

To prove this we first show that there exists $a>0$ such that

$$
\left|\psi\left(z_{k}+\zeta\right)\right| \geq a \text { whenever }|\zeta|=1 \text { and } k=1,2, \ldots
$$

By assumption (4.6) the unit disks centered at the $z_{k}$ 's are disjoint so

$$
\min \left\{\left|\psi\left(z_{k}+\zeta\right)\right|: k=1,2,|\zeta|=1\right\}>0 .
$$

Fix $k \geq 3$ and observe that

$$
\psi\left(z_{k}+\zeta\right)=\left(1-\frac{z_{k}+\zeta}{z_{1}}\right) \prod_{j=2}^{k-1}\left(1-\frac{z_{k}+\zeta}{z_{j}}\right)\left(-\frac{\zeta}{z_{k}}\right) \prod_{j=k+1}^{\infty}\left(1-\frac{z_{k}+\zeta}{z_{j}}\right)
$$

We are going to find uniform (in $k$ ) estimates for terms appearing in the above equality. We have

$$
\begin{aligned}
\left|\left(1-\frac{z_{k}+\zeta}{z_{1}}\right)\left(-\frac{\zeta}{z_{k}}\right)\right| & \geq \frac{\left|z_{k}\right|-\left|z_{1}\right|-1}{\left|z_{1} z_{k}\right|} \\
& \geq \frac{1}{\left|z_{1}\right|}-\frac{1}{\left|z_{2}\right|}-\frac{1}{\left|z_{1} z_{2}\right|} \\
& >0 .
\end{aligned}
$$


The next term is estimated as follows

$$
\begin{aligned}
\left|\prod_{j=2}^{k-1}\left(1-\frac{z_{k}+\zeta}{z_{j}}\right)\right| & =\left|\prod_{j=1}^{k-2}\left(1-\frac{z_{k}}{z_{k-j}}-\frac{\zeta}{z_{k-j}}\right)\right| \\
& \geq \prod_{j=1}^{k-2}\left(\left|\frac{z_{k}}{z_{k-j}}\right|-\frac{1}{\left|z_{k-j}\right|}-1\right) \\
& \geq \prod_{j=1}^{k-2}\left(\lambda^{j}-\frac{1}{\left|z_{1}\right|}-1\right)
\end{aligned}
$$

The last term tends to infinity as $k \rightarrow \infty$. By (4.5) we see that it is always positive, so there exists a uniform positive lower bound for all $k$ 's. We now turn to the remaining part:

$$
\begin{aligned}
\left|\prod_{j=k+1}^{\infty}\left(1-\frac{z_{k}+\zeta}{z_{j}}\right)\right| & =\prod_{j=1}^{\infty}\left|1-\frac{z_{k}+\zeta}{z_{k+j}}\right| \\
& \geq \prod_{j=1}^{\infty}\left(1-\frac{\left|z_{k}\right|+1}{\lambda^{j}\left|z_{k}\right|}\right) \\
& =\prod_{j=1}^{j_{0}}\left(1-\frac{\left|z_{k}\right|+1}{\lambda^{j}\left|z_{k}\right|}\right) \prod_{j=j_{0}+1}^{\infty}\left(1-\frac{\left|z_{k}\right|+1}{\lambda^{j}\left|z_{k}\right|}\right),
\end{aligned}
$$

where $j_{0}$ is the minimal integer for which $1-\frac{2}{\lambda^{j}}>0$ for $j \geq j_{0}$. Hence

$$
\prod_{j=j_{0}+1}^{\infty}\left(1-\frac{\left|z_{k}\right|+1}{\lambda^{j}\left|z_{k}\right|}\right) \geq \prod_{j=j_{0}+1}^{\infty}\left(1-\frac{2}{\lambda^{j}}\right)>0
$$

To complete the proof of (4.8) we proceed to show that

$$
1-\frac{\left|z_{k}\right|+1}{\lambda^{j}\left|z_{k}\right|}>\tilde{c}, \quad j=1, \ldots, j_{0},
$$

with some $\tilde{c}>0$. Indeed,

$$
1-\frac{\left|z_{k}\right|+1}{\lambda^{j}\left|z_{k}\right|} \geq 1-\frac{\left|z_{k}\right|+1}{\lambda\left|z_{k}\right|}=1-\frac{1}{\lambda}-\frac{1}{\lambda\left|z_{k}\right|} \geq 1-\frac{1}{\lambda}-\frac{1}{\lambda\left|z_{2}\right|},
$$

which implies (4.9). So we have proved (4.8). 
To deduce (4.7) from (4.8) we need to apply the Wiman theorem stating that for an entire function $f: \mathrm{C} \rightarrow \mathrm{C}$ of sufficiently small growth (i.e. dominated by $e^{\alpha|z|^{\beta}}$ with some $\alpha>0$ and $\left.\beta \in\left(0, \frac{1}{2}\right)\right)$ there exists a strictly increasing sequence of radii $\left\{R_{j}\right\}_{j=1}^{\infty}$ such that $R_{j} \rightarrow \infty$ and $\min \left\{|f(z)|:|z|=R_{j}\right\} \rightarrow \infty$ when $j \rightarrow \infty$ (cf. [2, Thm 3.1.1]). In particular, we are able to find a sequence $\left\{R_{j}\right\}_{j=1}^{\infty}$ satisfying $\min \left\{|\psi(z)|:|z|=R_{j}, j \geq 1\right\} \geq a$, where $a$ is a constant appearing in (4.8). In order to prove (4.7) it suffices to notice that

$$
|\psi(z)| \geq a, \quad z \in \Delta\left(0, R_{j}\right) \backslash \bigcup_{k=1}^{\infty} \Delta\left(z_{k}, 1\right)
$$

for each $j \geq 1$. This in turn follows from the minimum principle applied to $\psi$ restricted to the interior of $\Delta\left(0, R_{j}\right) \backslash \bigcup_{k=1}^{\infty} \Delta\left(z_{k}, 1\right)$. So the property (4.7) is proved.

Having established (4.7) we are in a position to finish the proof. Suppose that $F: \mathrm{C} \rightarrow \mathrm{C}$ is entire and chosen so that $\frac{F}{\psi}$ is again entire. Applying (2.2) and (4.7) we obtain

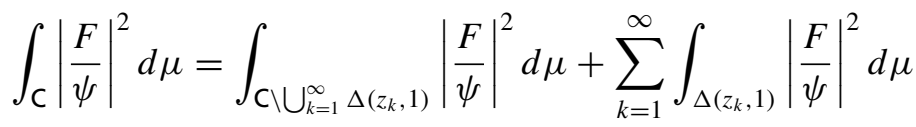

$$
\begin{aligned}
& \leq \int_{C \backslash \bigcup_{k=1}^{\infty} \Delta\left(z_{k}, 1\right)}\left|\frac{F}{\psi}\right|^{2} d \mu+e^{3} \sum_{k=1}^{\infty} \int_{\Delta\left(z_{k}, 2\right) \backslash \Delta\left(z_{k}, 1\right)}\left|\frac{F}{\psi}\right|^{2} d \mu \\
& \leq \frac{1}{a^{2}} \int_{C \backslash \bigcup_{k=1}^{\infty} \Delta\left(z_{k}, 1\right)}|F|^{2} d \mu+\frac{e^{3}}{a^{2}} \sum_{k=1}^{\infty} \int_{\Delta\left(z_{k}, 2\right) \backslash \Delta\left(z_{k}, 1\right)}|F|^{2} d \mu \\
& =\frac{1}{a^{2}} \int_{C \backslash \bigcup_{k=1}^{\infty} \Delta\left(z_{k}, 1\right)}|F|^{2} d \mu+\frac{e^{3}}{a^{2}} \int_{\bigcup_{k=1}^{\infty} \Delta\left(z_{k}, 2\right) \backslash \Delta\left(z_{k}, 1\right)}|F|^{2} d \mu \\
& \leq \frac{e^{3}+1}{a^{2}} \int_{C}|F|^{2} d \mu \text {. }
\end{aligned}
$$

This implies that $\left\|T_{\psi} f\right\|^{2} \geq a^{2}\left(e^{3}+1\right)^{-1}\|f\|^{2}$ for $f \in \mathscr{D}\left(T_{\psi}\right)$. The proof is complete.

We now proceed with the example described in the beginning of this section. Let $\left\{z_{k}\right\}_{k=1}^{\infty}$ be any sequence satisfying assumptions of Theorem 4.1 and such 
that $e^{z_{k}^{2} / 2}=1, k \geq 1$. Define the entire function $\varphi$ by

$$
\varphi(z):=\frac{e^{z^{2} / 2}-1}{\prod_{k=1}^{\infty}\left(1-\frac{z}{z_{k}}\right)} .
$$

In the next proposition we list some properties of the operator $T_{\varphi}$.

Proposition 4.2.

(i) $\mathscr{P} \subseteq \mathscr{D}\left(T_{\varphi}\right)$.

(ii) $\mathscr{P}$ is not dense in $\mathscr{D}\left(T_{\varphi}\right)$ with respect to the graph norm of $T_{\varphi}$.

(iii) $\mathscr{E} \nsubseteq \mathscr{D}\left(T_{\varphi}\right)$.

Proof. Throughout the proof we will use the notation: $r(z):=z$ and $\psi_{j}(z):=\prod_{k=j}^{\infty}\left(1-\frac{z}{z_{k}}\right), j \geq 1, z \in \mathrm{C}$.

(i) Given arbitrary $j \geq 1$ we show that $r^{j} \varphi \in \mathscr{B}$. First observe that

$$
\left|\frac{z^{j}\left(e^{z^{2} / 2}-1\right)}{\prod_{k=1}^{j+2}\left(1-\frac{z}{z_{k}}\right)}\right| \leq C \frac{e^{|z|^{2} / 2}}{(|z|+1)^{2}}, \quad z \in \mathrm{C},
$$

with a properly chosen $C>0$. Since the right hand side of this inequality is square summable with respect to $\mu$ we infer that the function $r^{j} \varphi \psi_{j+3}$ belongs to $\mathscr{B}$. Applying Theorem 4.1 to $\psi_{j+3}$ yields

$$
\left\|r^{j} \varphi \psi_{j+3}\right\| \geq c\left\|r^{j} \varphi\right\|
$$

with some $c>0$. So $r^{j} \in \mathscr{D}\left(T_{\varphi}\right)$.

(ii) We claim that the function $g(z):=e^{-z^{2} / 4}$ belongs to the domain of $T_{\varphi}$ but it cannot be approximated by polynomials with respect to the induced graph norm. Indeed, $g \in \mathscr{D}\left(T_{\varphi}\right)$, because $\left(g \varphi \psi_{1}\right)(z)=e^{z^{2} / 4}-e^{-z^{2} / 4}$ belongs to $\mathscr{B}$ and

$$
\left\|g \varphi \psi_{1}\right\| \geq c\|g \varphi\|
$$

with some $c>0$ obtained in virtue of Theorem 4.1.

We are now going to disprove the possibility of approximating $g$ by polynomials in the graph norm of $T_{\varphi}$. Suppose, contrary to our claim, that one can find a sequence $\left\{p_{k}\right\}_{k=1}^{\infty}$ such that $p_{k} \rightarrow g$ and $p_{k} \varphi \rightarrow g \varphi, k \rightarrow \infty$. We have

$$
\left|p_{k}(z) \varphi(z)\right|=\left|\left\langle p_{k} \varphi, e_{z}\right\rangle\right| \leq\left\|p_{k} \varphi\right\|\left\|e_{z}\right\|, \quad z \in \mathrm{C}, k \geq 1 .
$$

Since the sequence $\left\{p_{k} \varphi\right\}_{k=1}^{\infty}$ is bounded in $\mathscr{B}$, we deduce that

$$
\left|p_{k}(z) \varphi(z)\right| \leq D e^{|z|^{2} / 2}, \quad z \in \mathrm{C}, k \geq 1,
$$


with some constant $D>0$. Taking $z=x \in \mathrm{R}$ we get

$$
\left|p_{k}(x) \varphi(x)\right| \leq D e^{x^{2} / 2}, \quad x \in \mathrm{R}, k \geq 1,
$$

which together with uniform boundedness of the sequence $\left\{p_{k}\right\}_{k=1}^{\infty}$ near the origin (this follows from convergence in $\mathscr{B}$ of the sequence) implies that

$$
\left|p_{k}(x)\right| \leq D_{1}\left|\psi_{1}(x)\right|, \quad x \in \mathrm{R}, k \geq 1 .
$$

with a new constant $D_{1}>0$. Consider the function $h_{k}(z):=p_{k}(z) e^{-\sqrt[4]{z}}$ defined in $\{z \in C: \operatorname{Im} z \geq 0\}$, where the branch is chosen so that $\sqrt[4]{z} \in[0, \infty)$ for $z \in[0, \infty)$ and $\sqrt[4]{z} \in\{a(1+i): a \in[0, \infty)\}$ for $z \in(-\infty, 0]$. Observe that every $h_{k}$ is bounded on the real line. Indeed, this is a consequence of the following inequality

$$
\left|h_{k}(x)\right| \leq D_{1} e^{-\sqrt[4]{|x|} / 2}\left|\psi_{1}(x)\right|, \quad x \in \mathrm{R}, k \geq 1,
$$

and the fact that $\psi_{1}(z)$ is dominated by every function of the form $e^{A|z|^{\alpha}}$ with $A>0$ and $\alpha \in(0,1)$. Since $h_{k}$ is continuous in $\{z \in \mathrm{C}: \operatorname{Im} z \geq 0\}$, analytic in the interior of this set and bounded by $e^{A \sqrt{|z|}}$ with some $A>0$, we can apply the Phragmen-Lindelöf principle (cf. [2, Thm. 6.2.4]) to deduce that

$$
\left|h_{k}(z)\right| \leq D_{2}, \quad \operatorname{Im} z>0, k \geq 1,
$$

where $D_{2}:=\sup \left\{D_{1} e^{-\sqrt[4]{|x|} / 2}\left|\psi_{1}(x)\right|: x \in \mathrm{R}\right\}$. This means that

$$
\left|p_{k}(z)\right| \leq D_{2}\left|e^{\sqrt[4]{z}}\right|, \quad \operatorname{Im} z>0, k \geq 1,
$$

which is in contradiction with the convergence $p_{k}(z) \rightarrow e^{-z^{2} / 4}, k \rightarrow \infty$.

(iii) We will check that $e_{a} \notin \mathscr{D}\left(T_{\varphi}\right)$ when $\operatorname{Re} a>0$. Suppose that $e_{a} \in$ $\mathscr{D}\left(T_{\varphi}\right)$, then

$$
\left|e_{a}(z) \varphi(z)\right|=\left|\left\langle e_{a} \varphi, e_{z}\right\rangle\right| \leq\left\|e_{a} \varphi\right\| e^{|z|^{2} / 2}, \quad z \in \mathrm{C} .
$$

It follows that

$$
\left|e^{x \bar{a}}\left(e^{x^{2} / 2}-1\right)\right| \leq\left\|e_{a} \varphi\right\| e^{x^{2} / 2}\left|\psi_{1}(x)\right|, \quad x \in \mathrm{R},
$$

which is a contradiction, since $\left|\psi_{1}(x)\right|$ is dominated by $e^{\sqrt{|x|}}$ on the real axis. The proof is complete.

Remark 4.3. It is known that if $P$ is any polynomial satisfying

$$
|P(x)| \leq M(x), \quad x \in \mathrm{R},
$$


where

$$
\int_{-\infty}^{+\infty} \frac{\log ^{+} M(x)}{1+x^{2}} d x<+\infty
$$

then a majorant of exponential growth in $|z|$ can be given for $|P(z)|$ in the whole complex plane (the condition (4.10) is sharp). However, instead of just quoting this theorem, we preferred to give an ad hoc proof for (ii), for the reader's convenience.

The above example was found by the second named author as an attempt to find a "constructive" approach to a result published in [3] (Theorem 2.2), proof of which required existence of an entire function $\varphi$ such that it belongs to $\mathscr{B}$ with all polynomial multiples, yet some exponential multiple does not belong to $\mathscr{B}$. Later on, it occurred that this function is also a good example for disproving the polynomial approximation property as it is stated in Proposition 4.2(ii). This in turn may be regarded as a contribution to the topic of paper [1], which deals with polynomial approximation in the Segal-Bargmann type spaces. However, it would be far more significant to establish whether there exists an entire function $\varphi$ satisfying conditions (i) and (ii) of Proposition 4.2 together with $\mathscr{E} \subseteq \mathscr{D}\left(T_{\varphi}\right)$. In this way we would get the answer to the following open problem: is $\Pi_{\varphi}$ always adjoint to $T_{\varphi}$ with $\varphi: \mathrm{C} \rightarrow \mathrm{C}$ entire and such that $\varphi e_{z} \in \mathscr{B}$ for all $z \in C$ ?

\section{REFERENCES}

1. Borichev, A. A., The polynomial approximation property in Fock-type spaces, Math. Scand. 82 (1998), 256-264.

2. Boas, R. P., Entire Functions, Academic Press, New York 1954.

3. Cichoń, D., Notes on unbounded Toeplitz operators in Segal-Bargmann spaces, Ann. Polon. Math. 3 (1996), 227-235.

4. Cichon, D., Generalization of the Newman-Shapiro isometry theorem and Toeplitz operators, Integral Equations Operator Theory 34 (1999), 414-438.

5. Ehrenpreis, L., Fourier Analysis in Several Complex Variables, Pure Appl. Math. vol. XVIII, 1970.

6. Gel'fond, A. O., The Calculus of Finite Differences, Izdat. "Nauka”, Moscow 1967 (Russian ed.).

7. Janas, J., Toeplitz and Hankel operators on Bargmann spaces, Glasgow Math. J. 30 (1988), 315-323.

8. Janas, J., Unbounded Toeplitz operators in the Bargmann-Segal space, Studia Math. 99(1991), 87-99.

9. Janas, J., and Stochel, J., Unbounded Toeplitz operators in the Segal-Bargmann space. II, J. Funct. Anal. 126 (1994), 418-447.

10. Newman, D. J. and Shapiro, H. S., Fischer spaces of entire functions, Proc. Sympos. Pure Math. XI, "Entire functions and related parts of analysis", ed. J. Korevaar (1968), 360-369. 
11. Newman, D. J., and Shapiro, H. S., Certain Hilbert spaces of entire functions, Bull. Amer. Math. Soc. 72 (1966), 971-977.

\author{
INSTYTUT MATEMATYKI \\ UNIWERSYTET JAGIELLOŃSKI \\ UL. REYMONTA 4 \\ 30-059 KRAKÓW \\ POLAND \\ E-mail: cichon@im.uj.edu.pl
}

DEPARTMENT OF MATHEMATICS

KUNGL TEKNISKA HÖGSKOLAN

10044 STOCKHOLM

SWEDEN

E-mail: shapiro@math.kth.se 